\title{
FERROELECTRIC PLASMA SOURCE FOR HEAVY ION BEAM CHARGE NEUTRALIZATION *
}

\author{
Philip C. Efthimion, Erik P. Gilson, Larry Grisham and Ronald C. Davidson \\ Plasma Physics Laboratory, Princeton University, Princeton, NJ, 08543 USA \\ Simon Yu, William Waldron and B. Grant Logan, Lawrence Berkeley National Laboratory \\ University of California, Berkeley, CA, 94720 USA
}

\begin{abstract}
Plasmas are employed as a source of unbound electrons for charge neutralizing heavy ion beams to allow them to focus to a small spot size. Calculations suggest that plasma at a density of 1-100 times the ion beam density and at a length $\sim 0.1-1 \mathrm{~m}$ would be suitable. To produce one-meter plasma, large-volume plasma sources based upon ferroelectric ceramics are being developed. These sources have the advantage of being able to increase the length of the plasma and operate at low neutral pressures. The source utilizes the ferroelectric ceramic $\mathrm{BaTiO}_{3}$ to form metal plasma. The drift tube inner surface of the Neutralized Drift Compression Experiment (NDCX) will be covered with ceramic, and high voltage $(\sim 1-5 \mathrm{kV})$ applied between the drift tube and the front surface of the ceramic by placing a wire grid on the front surface. A prototype ferroelectric source $20 \mathrm{~cm}$ long has produced plasma densities of $5 \times 10^{11} \mathrm{~cm}^{-3}$. The source was integrated into the previous Neutralized Transport Experiment (NTX), and successfully charge neutralized the $\mathrm{K}^{+}$ion beam. Presently, the one-meter source is being fabricated. The source is being characterized and will be integrated into NDCX for charge neutralization experiments.
\end{abstract}

\section{INTRODUCTION}

HIBALL-II represents a possible heavy ion fusion reactor design [1]. The final focusing magnets must focus multiple heavy ion beams to a small spot size in the target chamber. This will require a deliberate charge neutralization of the ion beams. The Neutralized Transport Experiment (NTX) [2] has been configured to investigate the most promising neutralization methods. One charge neutralization concept employs a heavy ion beam propagating through a highly ionized plasma column [3]. The plasma will be created or injected into the drift section between the last magnetic lens and the reactor chamber. The cold plasma ion motion is neglected, and electrons from the cylindrical plasma move into the beam channel, reducing the net positive beam charge over the larger volume of the plasma channel. Ion beam densities are in the range of $10^{8}-10^{9} \mathrm{~cm}^{-3}$ for NTX. Present calculations require the plasma to be in the range of 0.1- 2 meters in length with an electron density comparable to 1-100 times the beam density. The operating pressure for the plasma needs to be in the range of $10^{-6}-10^{-5}$ Torr to prevent neutrals from stripping the beam ions to higher charge states. New experiments to explore compressing the bunch length axially have begun on NDCX and also require a one-meter-long plasma source.

\section{FERROELECTRIC PLASMA SOURCE}

Ferroelectric materials have been intensively examined as high current density electron emitters [5-7]. They have been projected to serve as large-surface-area, highcurrent-density cathodes. An electrode mesh-like structure is mounted to the emitting side of the ferroelectric material and the back surface has a metal plate electrode. A $3-15 \mathrm{kV}$ potential is applied to the electrodes depending upon the thickness of the ferroelectric material. For ultra-thin-film ferroelectric materials, the applied voltage results in spontaneous polarization reversal on a nanosecond timescale, and a high electric field. Spontaneous polarization reversal yields a noncompensated charge at the surface and a high electron emission across the entire thin film.

For millimeter-thick ferroelectrics, the electric fields are too small to produce polarization reversal. However, plasma emission is observed and is produced by electron emission from the vacuum micro-gaps between the dielectric surface and the edge of the metal electrode surface [8]. For this configuration, the value of the dielectric constant is the key factor. Commonly used ferroelectric materials have extremely large dielectric constants: $\mathrm{BaTiO}_{3}$ has a dielectric constant in the range of 1000-3000, and $\mathrm{Pb}(\mathrm{Zr}, \mathrm{Ti}) \mathrm{O}_{3}$ (PZT) has a dielectric constant in the range of 3000-6000. Once the threshold voltage is reached, plasma is formed over the entire surface of the dielectric. Typical current density yields are $0.5 \mathrm{~A} / \mathrm{cm}^{2}$. Plasma emission from these dielectrics has been characterized [8]. Typically, $8-16 \mathrm{kV}, 0.25 \mu \mathrm{s}$ pulses are applied to the electrodes at operating pressures near $10^{-5}$ Torr. The fact that the plasma is essentially all metal means that neutrals stick to the walls of the vacuum system and do not result in a pressure rise.

The characteristics of this plasma source are exactly what are required for the charge neutralization experiments on NTX and the beam compression experiments on NDCX. Furthermore, its ability to make the plasma-emitting layer arbitrarily long is important for the one-meter-long plasma source. The source will be mounted on the walls of the drift tube just past the last focusing quadrapole 
magnet. The drift tube is approximately 3 inches in diameter. This small tube diameter will allow the density to be in the $10^{12} \mathrm{~cm}^{-3}$ range on axis. The approach taken is to build a source with cylindrical ferroelectric pieces stacked together to form a 1-m-long ferroelectric cylinder. Initially, a 20 -cm-long ferroelectric source made of 1 " long and $1 / 4$ " thick ferroelectric cylinders was built for evaluation (Fig. 1). The front surface electrode was made of 36, 10 mil. stainless steel wires strung along the length of the cylinder. The wires are mounted at each end of the source with an aluminium ring with 36 set screws. Each ring is mounted in a Delrin insulating sleeve to isolate it from the outer surface of the ferroelectric cylinders. The wires are pulled taught and actually hold the ferroelectric cylinders firmly together. Figure 1 clearly shows the wires mounted on one of the aluminium rings and the black Delrin insulating sleeve behind the rings. Also shown in the photograph is the copper wire that is wound around the outer surface of the ferroelectric cylinder to provide a good electrical contact. Electrically, the high-voltage pulse is applied to one of the aluminium mounting rings and the copper wire wound around the outer surface of the cylinder. All of the stainless steel wires are at the same potential through the aluminium rings at each end of the source.

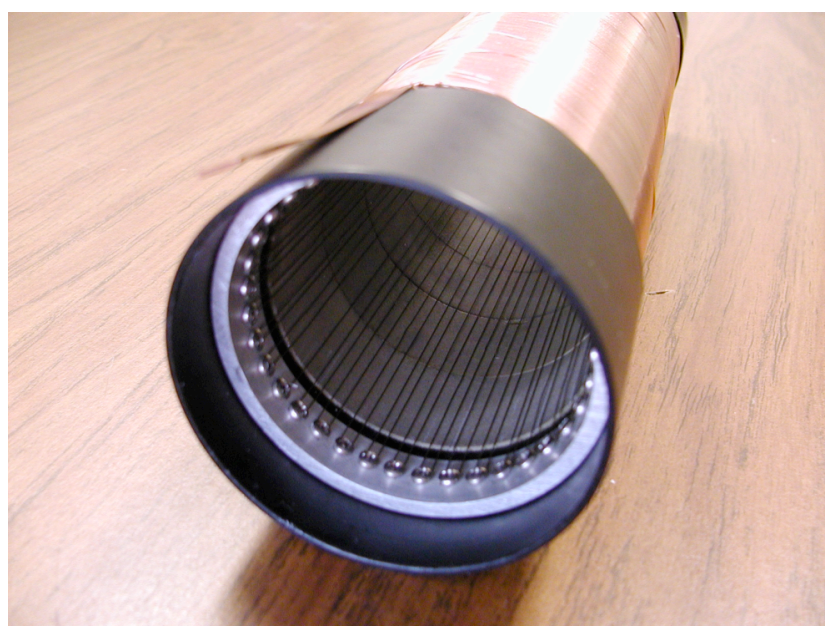

Figure 1: Photograph of PZT ferroelectric plasma source.

The power supply for this pulsed source is a standard capacitor bank with a pulse-forming network to match the impedance of the source and maintain the microsecond pulse shape. As presently configured the pulse-forming network is matched to $4 \mathrm{ohms}$ and has a maximum output of $8 \mathrm{kV}$ and $2 \mathrm{kA}$. Thyratrons control the discharge of the charging capacitors. The output of the power supply is two 1-microsecond pulses with an adjustable time delay between the pulses. For the 15-cm-long PZT ferroelectric source, the typical operating parameters are $6 \mathrm{kV}$ and 800 amperes. Figure 2 is a photograph of the operating ferroelectric source, showing numerous localized discharges adjacent to the inner electrode wires (the view is blocked by the high-voltage feed-through). Langmuir probe measurements along the axis of the source show a somewhat constant density inside the cylindrical source. Since the source is pulsed, the electron density and temperature decays over $50 \mu \mathrm{s}$. Figure 3 shows a profile of the electron density and temperature at times $10 \mu \mathrm{s}$

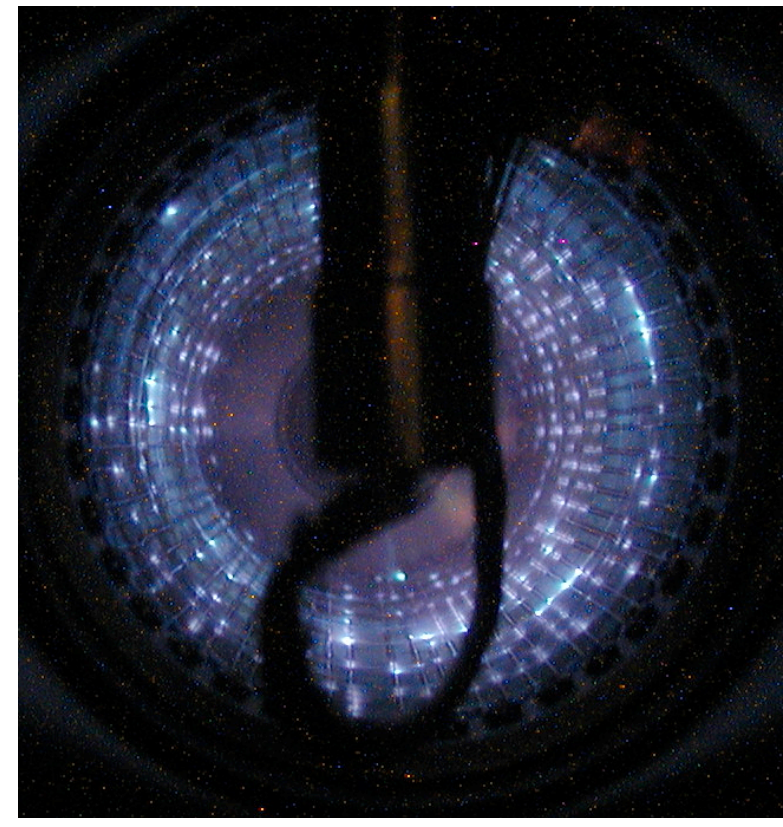

Figure 2: Photograph of operating plasma source with numerous plasma discharges adjacent to the wires.

and $35 \mu \mathrm{s}$ after the high-voltage pulse. The density is in the range of $10^{10}-10^{12} \mathrm{~cm}^{-3}$ at temperatures of 5 and 10 $\mathrm{eV}$, respectively. With an ion beam density in NTX of 1 x $10^{8} \mathrm{~cm}^{-3}$, the conditions in the ferroelectric plasma source are sufficient to neutralize the ion beam space charge. Consequently, the plasma conditions can be controlled by adjusting either the applied high voltage or the delay when the ion beam passes through the plasma. With the ion beam pulse only $1 \mu$ s long, it is easy to control the plasma conditions by adjusting the time when the beam pulse passes through the plasma.

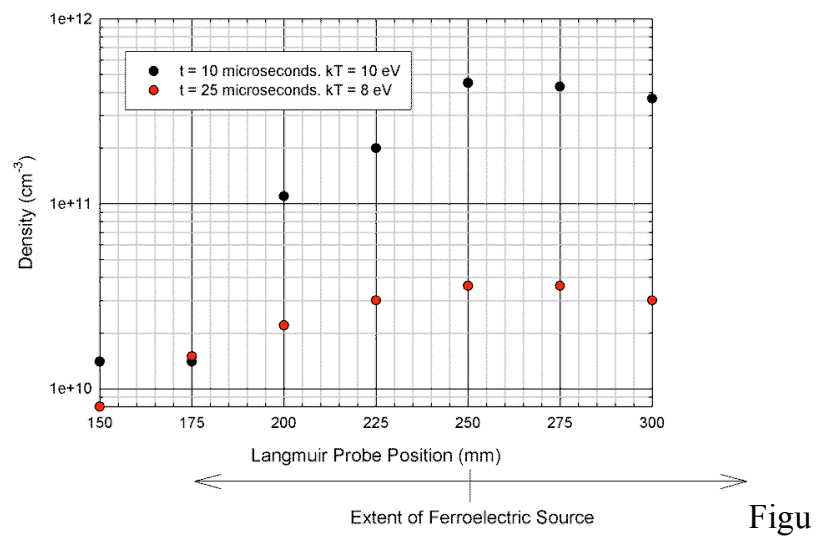

re 3: Langmuir probe measurements along the axis of the PZT ferroelectric source. 


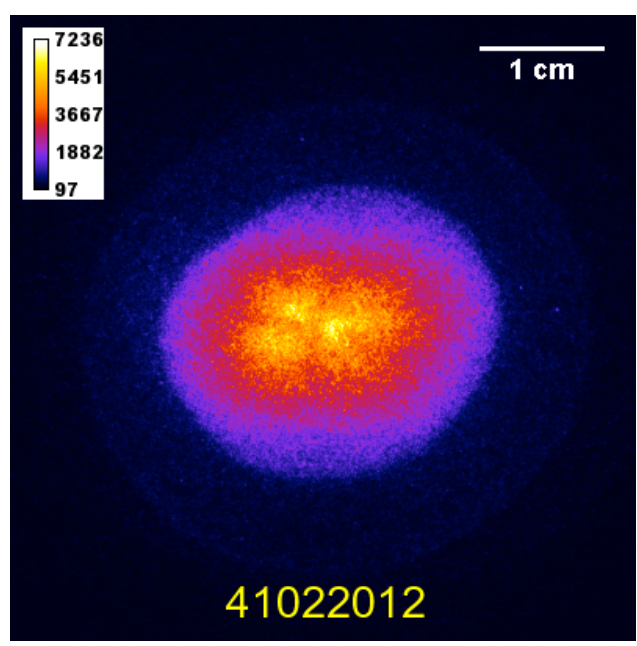

Before Neutralization

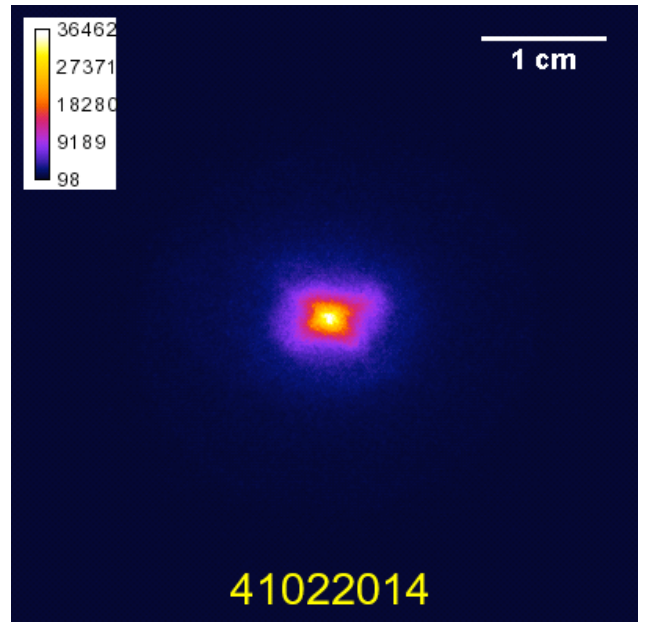

After Neutralization

Figure 4: Images of the beam spot size before and after neutralization.

\section{NEUTRALIZATION EXPERIMENTS ON NTX}

The ferroelectric source was then shipped to LBNL to participate in charge neutralization experiments on NTX. In the experiments, the last focusing quadrapole magnets direct the beam on a trajectory that focuses it to a spot size smaller than permitted by the space-charge limit. Before the beam approaches the space-charge limit, it enters the plasma created by the ferroelectric source, where it accumulates electrons and charge-neutralizes. The neutralized beam continues on its focusing trajectory, does not diverge, and achieves an extremely small spot size. Figure 4 has images of the beam before and after the neutralization. With the plasma source turned on, the beam achieves a spot size smaller than achievable without charge neutralization.

The spot size was examined during a parameter scan of the source discharge voltage. The Langmuir probe measurements indicate that the source discharge current is proportional to the plasma electron density. In the scan, while the current increased the beam spot size decreased. Consequently, the scan shows that as the plasma density increases the spot size decreases, consistent with charge neutralization theory and simulations.

\section{FUTURE PLANS}

The results from the PZT prototype ferroelectric source are sufficient to proceed with the design and building of the 1-meter-long plasma source for NDCX. It is being fabricated with $\mathrm{BaTiO}_{3}$ ceramic instead of the PZT ceramic used in the plasma source for NTX. The first section of the source has been fabricated and successfully tested. The remaining sections of the source are currently being fabricated. On NDCX, a velocity tilt will be imposed on the beam pulse to compress the beam axially. In addition, the beam will also be radially focused as was done on NTX. The compression of the beam can be as large as a factor of 100 . To achieve this requires the 1meter-long plasma source to neutralize the beam as it completes its compression and focusing.

\section{REFERENCES}

[1] B. Badger, et al. (1984), "HIBALL-II, An Improved Conceptual Heavy Ion Beam Driven Fusion Reactor Study", KfK-3480, Kernforschungszentrum Karlsruhe Report.

[2] P. K. Roy, S. Yu, et al., Phys. Plasmas 11, 2890 (2004).

[3] B. G. Logan and D. A. Callahan, Nucl. Instr. and Meth. In Phys. Research A 415, 468 (1998).

[4] P. C. Efthimion, et al., Nucl. Instr. and Meth. in Phys. Research A, in press.

[5] G. Rosenman, et al., Appl. Phys. Rev. 88, 6109 (2000).

[6] A. Dunaevsky, et al., Journal of Applied Physics 95, 4621 (2004).

[7] A. Dunaevsky, et al., Journal of Applied Physics 90, 3689 (2001).

[8] A. Dunaevsky, et al., Journal of Applied Physics 89, 4480 (2001). 